MATEC Web of Conferences 44, 01092 (2016)

DOI: $10.1051 /$ matecconf/20164401092

(C) Owned by the authors, published by EDP Sciences, 2016

\title{
The Study and Application of "Trinity System" Based on Multi-living Agent Method
}

\author{
Dong Zhong ${ }^{1, a}$, Zhong Ming Li', Chun Chen ${ }^{2}$, Ruo Lin Ruan ${ }^{3}$ \\ ${ }^{1}$ College of electronic and information, Hubei University of Science and Technology, Xianning, China \\ ${ }^{2}$ College of Physical Education, Hubei University of Science and Technology, Xianning, China \\ ${ }^{3}$ College of biomedical engineering, Hubei University of Science and Technology, Xianning, China
}

\begin{abstract}
Trinity System", which is consisted of "110,122,119" three police command stations merging into a police command center, the system is to achieve a unified alarm, unified command, joint action and to provide emergency services and urban public security. Multi-living Agent Method is a new method to analysis the complex system. This paper proposes to use a new method of multi-living agent to design "Trinity System" based on analyzing the traditional "Trinity System", according to the guiding theory of the system. The paper designs the whole framework and system software architecture of "Trinity System". It provides a good research direction to enhance the capabilities of emergency response for "Trinity System".
\end{abstract}

\section{Introduction}

With the rapid development of the economy and society, the city public security has become an increasing important issue. Because urban disasters and emergencies can not be avoided completely and a single sector of the government subordinate agencies is difficult to deal with the disasters and emergencies, meanwhile solving the memory and distinguish of special service code, we need to build emergency response mechanism. The key problem is to achieve "110,122,119" three police command units merging into a police command center, this is the "Trinity System". It can achieve a unified alarm, unified command, joint action to ensure emergency services and city public security. "Trinity System" ${ }^{[1]}$ is an integrated system, which is consists of several subsystems. Meanwhile, "Trinity System" is a complex information system. This paper proposes to use a new method of multi-living agent to design "Trinity System" based on analyzing the traditional "Trinity System", according to the guiding theory of the system. The paper designs the whole framework and system software architecture of "Trinity System", it provides a good research direction to enhance the capabilities of emergency response for "Trinity System".

2 The basic discussion of "Trinity System", the interposing of "Multi-living Agent" method

\section{1 "Trinity System" is Related closely to and}

\footnotetext{
a e-mail: zongdong0129@163.com
} 


\section{System Theory}

In addition to the general system characteristics, "Trinity System" has the characteristic of modular hierarchical system architecture design, the system software core is based on a unified messaging service platform, it merges independent species $(110,119,122)$ departments, multiple data, various business into a system, it can select the number of subsystems according to the actual need ${ }^{[2]}$.

The core principles of system theories, such as the movement of the formation about open dissipative system layer self-organizing mechanism, evolution of the system and environment, and so on. These system theories summarized highly the trend of the dynamic development. They have practical significance for the study of "Trinity System" [3]. For example, the improving of the police efficiency is related with the response alarm systems, the environment of command and control system, a unified monitoring and management platform. It requires the system and environment co-evolution to achieve our expected requirements. In this case, In this case, this paper proposes the new method of multi-living agent to analyze and study the whole framework and system software architecture. It has important significance to improve the emergency response capacity of "Trinity System".

\subsection{The basic Idea of "Multi-living Agent"}

In the first, the "living" in "Multi-living agent" only comes from the word of the function (action), then it became a specific technical word by re-define the specific examples after the application ${ }^{[4]}{ }^{[5]}$. It can be divided into three cases:

1) The word can be used only in a qualitative sense, which is corresponding with resting state or non-live (moving) of (inactivity);

2) The word has been defined as a measure of activity intensity. If it has been given the meaning of the activity, we should use the activation in the general; if the activity meaning has been removed, then we should use the non-activation or inactivation and other words;

3) To the physiological processes occurring in the organism or in active state or property; "Living" means a nature of activity for the vitality which is going on a tenacious.

"Living" gives the vigor to the system. It has showed the character of the survival and development for "Trinity System", it plays a important role in various functions. The "more" in "Multi-active agent" reflects the multiple functions in "Trinity System" [6] [7], it has the essential attributes of multiple location, multiple levels and a number of related subsystems, the "proxy " in "multi-living agent" integrated the tendency of the human, the system function, the system's basic structure and operation of the system based on" living "sense, mapping the future vision into reality system or a representative system, it also can be mapped to a specific system, namely "Trinity System", this system has the man-machine integration, scheduling on-site emergency response center and the combination of characteristics $^{[8]}$. This paper proposes the whole framework and system software architecture based on the above basic characteristics of multi-active agent.

\section{The design of the whole framework and system software architecture for "trinity system" based on multi-living agent method}

\subsection{The Design of the whole Framework for "Trinity System" based on Multi-living Agent}

This paper designs the whole framework for "Trinity System" based on multi-living agent, the system is constituted of a unified monitoring and management platform, the shared platform of the plans for management, GIS/GPS sharing platform, the exchange platform for information sharing, communication integration services platform, database sharing platform. This multi-agent system is mainly to provide daily communication support for the activity of the Public Order (110) Patrol (122), Fire (119) emergency services. In addition, it is to avoid duplication investment of the government, it also can provide daily scheduling tool for urban management, forest fire, flood and other government departments. Figure1 has shown the whole framework: 
For the whole framework of "Trinity System", this paper has a preliminary study based on multi-living agent approach, it is equivalent to using the multi-living agent approach to study a complex information system. For the "Trinity System", from the point of system theory, this is a complex information system and it is associated with assistant scheduling decision function, meanwhile it is a further high-level decision problem of remote dispatching. In this complex information system, "living" means in the top can be understood to make great effort to protect the network management and remote dispatching center to take the most efficient way to provide daily services for emergency communications support. At the same time, "living" also represents the timeliness and effectiveness measures of the communication when a problem comes from the system or communication handicaps occurs. "Multi-living" represents the functioning coordination among the various business systems platform agent layer (for example, response alarm system, information distribution system, a unified monitoring and management platform, GIS/GPS sharing platform, the exchange platform for information sharing, communication integration services platform, database, and various sub-sharing platform system). Meanwhile, under the construction of each "living agent", the agents can be divided into various sub-agents, such multi-level decomposition until a layer of specific hardware and software agents.

\subsection{The design of system software architecture for "Trinity System" based on Multi-living Agent}

The work model of "Trinity System" is a combined of unified alarm, unity and reunification alarm response alarm, the United Source police combination. The alarm command center dispatches directly to the fire command center, traffic police, patrol, police station and other police. Alarming unified platform is one of the main objectives of system implementation, Alarming platform software components are divided according to application level, we design the multi-living agent software system block diagram of "Trinity System" shown in Figure2:
The software system has been design in modular idea, the alarm system is consists of a unified platform services layer, support layer, the operating system layer, networking layer. The system should guarantee the access of the underlying, business processes and business systems are independent interfaces each other. From the viewpoint of system theories, this multi-living agent software architecture is also a complex information system. The system is mainly to achieve a unified response alarm, "110,122,119" merging into a unified response alarm platform. The paper divides the basic modules of the software system in accordance with the application of the software. It can ensure the independence among each module. The meaning of "living" in the multi-living agent software system can be understood to achieve the unity of response alarm, it can ensure the scheduling decision. At the same time, "living" also represents the business in case of alarm, the police intelligence information access interface, and police issued the interface, alarm forwarding interfaces and the external application interface are to ensure a unify platform software alarm information exchanging with the outside timeliness and effectiveness of the entire system to ensure data security. "Multi-living" represents the coordination and support of each business layer proxy of multi-agent software architecture (such as the access layer, interface layer, unified alarm platform services layer, unified platform support layer, the operating system level, network interconnection system layer, and so on.). The multi-living agent software architecture has the basic functions and features of following: Network connectivity layer proxy and the operating system layer proxy are the underlying software architecture of multi-living agent. It can ensure the system's security, stability and versatility. Support system layer proxy is running for the underlying application.

\section{Conclusions}

This paper proposes to use a new method of multi-living agent to design "Trinity System" based on analyzing the traditional "Trinity System", according to the guiding theory of the system. "Multi-living" represents the 
functioning coordination among the various business systems platform agent layer and system, through this, the whole framework has been designed in the paper. Meanwhile, software architecture of "Trinity System" including a unified platform services layer, support layer, the operating system layer, networking layer has been use the same method to research. It provides a good research direction to enhance the capabilities of emergency response for "Trinity System".

\section{Acknowledgements}

The authors gratefully acknowledge the financial support for this work provided by the education department of Hubei Province Science and technology research project, No. B2015077; the colleges and universities of Hubei Province in 2014 the Provincial College Students' innovation and entrepreneurship training plan, No. 201410927025 and 201410927029; The school educational reform key projects of Hubei University of Science and Technology, No. 2014-XA-007 and 2014-XC-025; The National Natural Science Foundation of China (NSFC) under the Grant No. 61271256 and 61575148; the Team Plans Program of the Outstanding Young Science and Technology Innovation of Colleges and Universities in Hubei Province, Grant No.T201513, the Program of the Natural Science Foundation of Hubei Province, Grant No. 2014BAA315 and No. 2015CFB452.

\section{References}

[1]. Arun Jotshi, Qiang Gong and Rajan Batta. Dispatching and Routing of Emergency Vehicles in Disaster Mitigation Using Data Fusion. Socio-Economic Planning Sciences, 43 pp 1-24, 2009

[2]. Ryan D. Murphy, Ben Torralva, Steven M and Yalisove. "The role of an interface on Ni film removal and surface roughness after irradiation by femtosecond laser pulses," Appl. Phys. Lett. 2014,102, 181602-181606
[3]. Yuanming Sun. "Solutions to the problems of urban emergency response system in China" (in Chinese), Chongqing Administration, No.5 2006,pp:75-77.

[4]. Haifeng Deng.Clean Development Mechanism right object of Energy Resources Law. Tsinghua University Research Center Web site . 2014.

[5]. Zhou Xie. Analysis on the Legal Property of the Certified Emission Reductions(CERs) in the project of CDM. 2014 International Conference on Education Reform and Management Innovation.

[6]. Yue Wang. The new method for the construction of complex information system - multiple active agents [J]. China engineer science, 2006, 8(5): 30-34.

[7]. ZhongDong, Chen chun. The study of scheduling function optimization for trunking communication system in CERS[J]. Science, technology and engineering, 2011, 18(3): 211-213.

[8]. Lin P,Lo S M,Ng W C.A GIS-Queue Model and Its Application to Regional Evacuation. Journal of Basic Science and Engineer- ing . 2015.

[9]. Atkey.D.A. Practical methodology for the measurement of screening effectiveness of trunking. IEEE Conference Publication n 464, 2008, pp: 77-83.

[10]. G L Stüber. Principles of Mobile Communication[M] . Kluwer Academic Publishers, Second Edition 2001.

[11]. Thompdon P A,Marchant E W.A computer model for the evacuation of large building populations. Fire Safety Journal . 2014.

[12]. Lai Hong Xiao, Cui Yingan, Cui Duwu. Call center architecture based on generic sound card $[\mathrm{J}]$. Computer Engineering, 2007, 22 (33):73-75.

[13]. Atkey.D.A. Practical methodology for the measurement of screening effectiveness of trunking.IEEE Conference Publication n 464, 2008, pp: 77-83. 


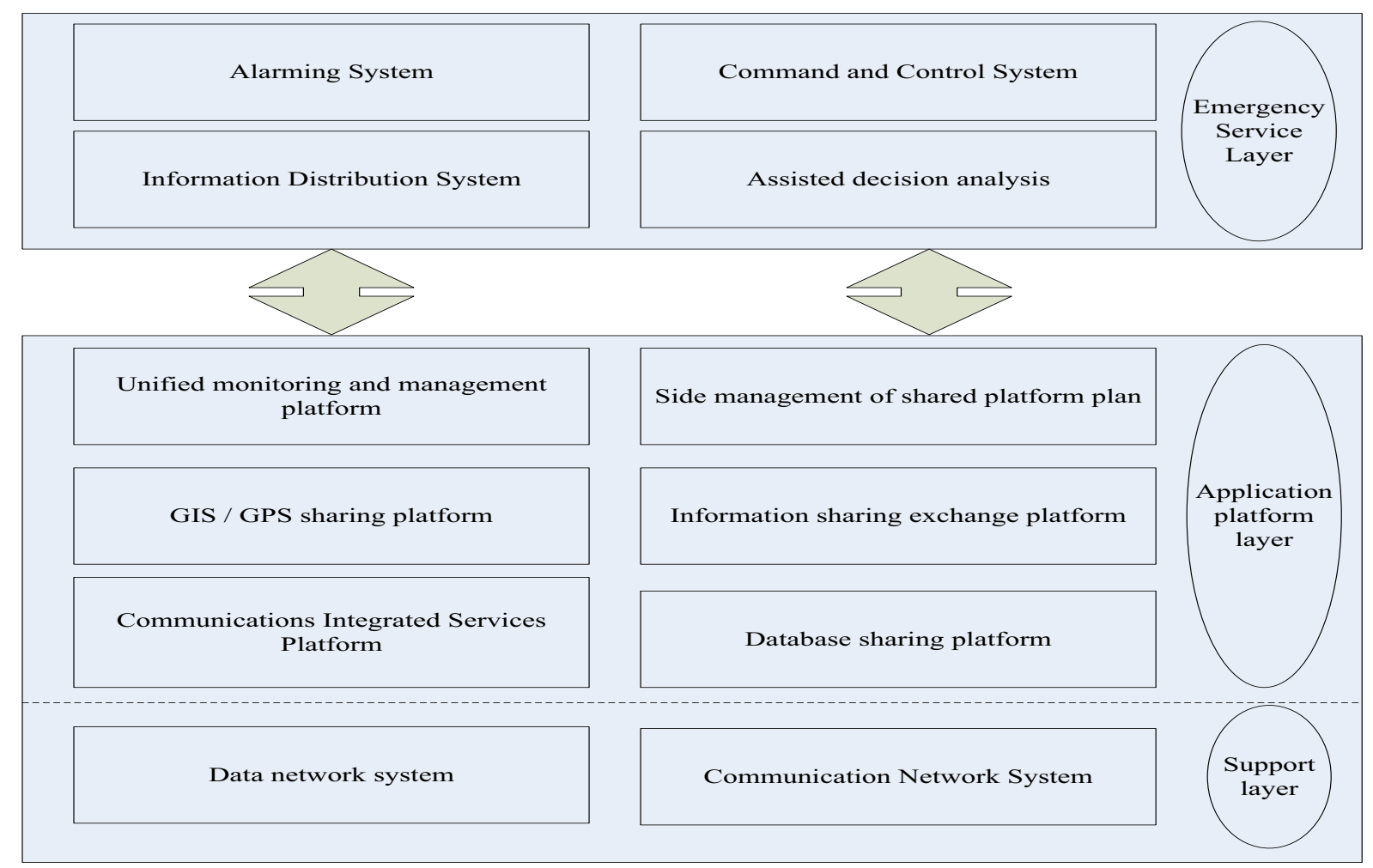

Figure1 Three more than one active agent system block diagram of the overall framework

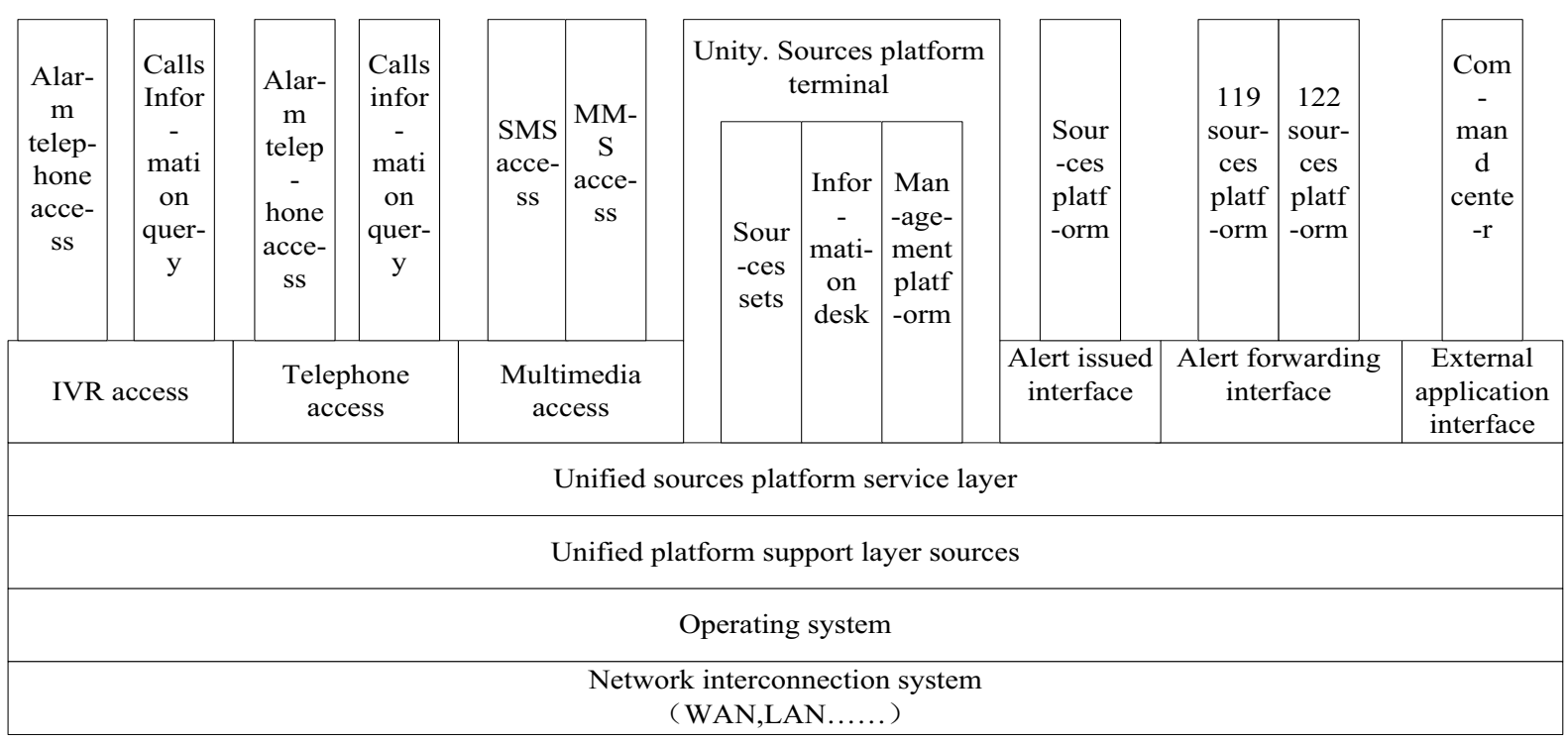

Figure2 Three more than one active agent software system block diagram

The first Author Introduction: Dong Zhong (1980--), Ph. D. His current research area is signal and communication system. 